\title{
Application of the product net technique and Kadec-Klee property to study nonlinear ergodic theorems and weak convergence theorems in uniformly convex multi-Banach spaces
}

\section{H.M. Kenari ${ }^{1}$, Reza Saadati ${ }^{*}$ (ID and Choonkil Park ${ }^{3}$}

*Correspondence: rsaadati@eml.cc ${ }^{2}$ Department of Mathematics, Iran University of Science and

Technology, Tehran, Iran Full list of author information is available at the end of the article

\begin{abstract}
Let $Y$ be a uniformly convex multi-Banach space which has not a Frechet differentiable norm. We use the technique of product net to obtain the nonlinear ergodic theorems in $Y$. Finally, let the dual of uniformly convex multi-Banach space have the Kadec-Klee property, we instate the weak convergence theorem in the case of reversible semi-group.
\end{abstract}

MSC: Primary 39A10; 39B72; secondary 47H10; 46B03

Keywords: Reversible semi-groups; Kadec-Klee property; Asymptotically nonexpansive mapping; Almost orbit; Uniformly convex multi-Banach space

\section{Preliminaries}

Dales and Polyakov in [1] introduced a multi-normed space by using the concept of operator sequence space, operator spaces, and Banach lattices; for more details and application, we refer to [1-3].

In this paper assume that $(Y,\|\cdot\|)$ is a complex normed space, and let $\ell \in \mathbb{N}$. We denote by $Y^{\ell}$ the vector space $Y \oplus \cdots \oplus Y$ consisting of $\ell$-tuples $\left(y_{1}, \ldots, y_{\ell}\right)$, where $y_{1}, \ldots, y_{\ell} \in Y$. The linear operations on $Y^{\ell}$ are defined coordinate-wise. The zero element of either $Y$ or $Y^{\ell}$ is denoted by 0 . We denote by $\mathbb{N}_{\ell}$ the set $\{1,2, \ldots, \ell\}$ and by $\Sigma_{\ell}$ the group of permutations on $\ell$ symbols.

Definition 1.1 Suppose that $Y$ is a vector space, and take $\ell \in \mathbb{N}$. For $\sigma \in \Sigma_{\ell}$, define

$$
B_{\sigma}(y)=\left(y_{\sigma(1)}, \ldots, y_{\sigma(\ell)}\right), \quad y=\left(y_{1}, \ldots, y_{\ell}\right) \in Y^{\ell} .
$$

For $\beta=\left(\beta_{j}\right) \in \mathbb{C}^{\ell}$, define

$$
K_{\beta}(y)=\left(\beta_{j} y_{j}\right), \quad y=\left(y_{1}, \ldots, y_{\ell}\right) \in Y^{\ell} .
$$

(c) The Author(s) 2019. This article is distributed under the terms of the Creative Commons Attribution 4.0 International License (http://creativecommons.org/licenses/by/4.0/), which permits unrestricted use, distribution, and reproduction in any medium, provided you give appropriate credit to the original author(s) and the source, provide a link to the Creative Commons license, and indicate if changes were made. 
Definition 1.2 Assume that $(Y,\|\cdot\|)$ is a complex (respectively, real) normed space, and take $m \in \mathbb{N}$. A multi-norm of level $m$ on $\left\{Y^{\ell}: \ell \in \mathbb{N}_{m}\right\}$ is a sequence $\left(\|\cdot\|_{\ell}: \ell \in \mathbb{N}_{m}\right)$ such that $\|\cdot\|$ is a norm on $Y^{\ell}$ for each $\ell \in \mathbb{N}_{m}$, such that $\|y\|_{1}=\|y\|$ for each $y \in Y$ (so that $\|\cdot\|_{1}$ is the initial norm), and such that the following Axioms (a1)-(a4) are satisfied for each $\ell \in \mathbb{N}_{m}$ with $k \geq 2$ :

(a1) for each $\sigma \in \Sigma_{\ell}$ and $y \in Y^{\ell}$, we have

$$
\left\|B_{\sigma}(y)\right\|_{\ell}=\|y\|_{\ell}
$$

(a2) for each $\beta_{1}, \ldots, \beta_{\ell} \in \mathbb{C}$ (respectively, each $\beta_{1}, \ldots, \beta_{\ell} \in \mathbb{R}$ ) and $y \in Y^{\ell}$, we have

$$
\left\|K_{\beta}(y)\right\|_{\ell} \leq\left(\max _{j \in \mathbb{N}_{\ell}}\left|\beta_{j}\right|\right)\|y\|_{\ell}
$$

(a3) for each $y_{1}, \ldots, y_{\ell-1}$, we have

$$
\left\|\left(y_{1}, \ldots, y_{\ell-1}, 0\right)\right\|_{\ell}=\left\|\left(y_{1}, \ldots, y_{\ell-1}\right)\right\|_{\ell-1}
$$

(a4) for each $y_{1}, \ldots, y_{\ell-1} \in Y$,

$$
\left\|\left(y_{1}, \ldots, y_{\ell-2}, y_{\ell-1}, y_{\ell-1}\right)\right\|_{\ell}=\left\|\left(y_{1}, \ldots, y_{\ell-1}\right)\right\|_{\ell-1} .
$$

In this case, $\left(\left(Y^{\ell},\|\cdot\|_{\ell}\right): \ell \in \mathbb{N}_{m}\right)$ is a multi-normed space of level $m$.

A multi-norm on $\left\{Y^{\ell}: \ell \in \mathbb{N}\right\}$ is a sequence

$$
\left(\|\cdot\|_{\ell}\right)=\left(\|\cdot\|_{\ell}: \ell \in \mathbb{N}\right)
$$

such that $\left(\|\cdot\|_{\ell}: \ell \in \mathbb{N}_{m}\right)$ is a multi-norm of level $m$ for each $m \in \mathbb{N}$. In this case, $\left(\left(Y^{m},\|\cdot\|_{m}\right): m \in \mathbb{N}\right)$ is a multi-normed space.

Lemma 1.3 ([3]) Let $\left(\left(Y^{\ell},\|\cdot\|_{\ell}\right): \ell \in \mathbb{N}\right)$ be a multi-normed space, and take $\ell \in \mathbb{N}_{m}$. Then

(a) $\|(y, \ldots, y)\|_{\ell}=\|y\|(y \in Y)$;

(b) $\max _{j \in \mathbb{N}_{\ell}}\left\|y_{j}\right\| \leq\left\|\left(y_{1}, \ldots, y_{\ell}\right)\right\|_{\ell} \leq \sum_{j=1}^{\ell}\left\|y_{j}\right\| \leq \ell \max _{j \in \mathbb{N}_{\ell}}\left\|y_{j}\right\|\left(y_{1}, \ldots, y_{\ell} \in Y\right)$.

It follows from (b) that if $(Y,\|\cdot\|)$ is a Banach space, then $\left(Y^{\ell},\|\cdot\|_{\ell}\right)$ is a Banach space for each $\ell \in \mathbb{N}$; in this case $\left(\left(Y^{\ell},\|\cdot\|_{\ell}\right): \ell \in \mathbb{N}\right)$ is a multi-Banach space.

Example 1.4 ([1]) The sequence $\left(\|\cdot\|_{\ell}: \ell \in \mathbb{N}\right)$ on $\left\{Y^{\ell}: \ell \in \mathbb{N}\right\}$ defined by

$$
\left\|\left(y_{1}, \ldots, y_{\ell}\right)\right\|_{\ell}:=\max _{j \in \mathbb{N}_{\ell}}\left\|y_{j}\right\| \quad\left(y_{1}, \ldots, y_{\ell} \in Y\right)
$$

is a multi-norm called the minimum multi-norm.

Example 1.5 ([1]) Assume that $\left\{\left(\|\cdot\|_{\ell}^{\beta}: \ell \in \mathbb{N}\right): \beta \in B\right\}$ is the (non-empty) family of all multi-norms on $\left\{Y^{\ell}: \ell \in \mathbb{N}\right\}$. For $\ell \in \mathbb{N}$, set

$$
\left\|\left(y_{1}, \ldots, y_{\ell}\right)\right\|_{k}:=\sup _{\beta \in B}\left\|\left(y_{1}, \ldots y_{\ell}\right)\right\|_{\ell}^{\beta} \quad\left(y_{1}, \ldots, y_{\ell} \in Y\right) .
$$

Then $\left(\|\cdot\|_{\ell}: \ell \in \mathbb{N}\right)$ is a multi-norm on $\left\{Y^{\ell}: \ell \in \mathbb{N}\right\}$, called the maximum multi-norm. 
By the property (b) of multi-norms and the triangle inequality for the norm $\|\cdot\|_{k}$, we can get the following properties. Suppose that $\left(\left(Y^{\ell},\|\cdot\|_{\ell}\right): \ell \in \mathbb{N}\right)$ is a multi-normed space. Let $\ell \in \mathbb{N}$ and $\left(y_{1}, \ldots, y_{\ell}\right) \in Y^{k}$. For every $i \in\{1, \ldots, \ell\}$, let $\left(y_{m}^{i}\right)_{m=1,2, \ldots}$ be a sequence in $Y$ such that $\lim _{m \rightarrow \infty} y_{m}^{i}=y_{i}$. Then for each $\left(z_{1}, \ldots, z_{\ell}\right) \in Y^{\ell}$ we have

$$
\lim _{m \rightarrow \infty}\left(y_{m}^{1}-z_{1}, \ldots, y_{m}^{\ell}-z_{\ell}\right)=\left(y_{1}-z_{1}, \ldots, y_{\ell}-z_{\ell}\right)
$$

A sequence $\left(y_{m}\right)$ in $Y$ is a multi-null sequence if, for every $\varepsilon>0$, there exists $m_{0} \in \mathbb{N}$ such that

$$
\sup _{\ell \in \mathbb{N}}\left\|\left(y_{n}, \ldots, y_{m+\ell-1}\right)\right\|_{\ell}<\varepsilon \quad\left(m \geq m_{0}\right) .
$$

Let $y \in Y$. We say that the sequence $\left(y_{m}\right)$ is multi-convergent to $y \in Y$ and write

$$
\lim _{m \rightarrow \infty} y_{m}=y
$$

when $\left(y_{m}-y\right)$ is a multi-null sequence.

Assume that $G$ is a semi-topological semi-group. In this article, $C$ is a nonempty bounded closed convex subset of a uniformly convex Banach space $X$. Let $X^{*}$ be the dual of $X$, then the value of $u^{*} \in X^{*}$ at $u \in X$ will be denoted by $\left\langle u, u^{*}\right\rangle$, and we associate the set

$$
J(u)=\left\{u^{*} \in X:\left\langle u, u^{*}\right\rangle=\|u\|^{2}=\left\|u^{*}\right\|^{2}\right\}
$$

It is clear from the Hahn-Banach theorem that $J(u)$ is not empty for all $u \in X$. Then the multi-valued operator $J: X \rightarrow X^{*}$ is called the normalized duality mapping of $X$, also $\Im_{k}=$ $\left\{J_{k}(t): t \in G\right\}$ is a reversible semigroup of asymptotically nonexpansive functions acting on $C$. Let $F\left(\Im_{k}\right)$ denote the set of all fixed points of $\Im_{k}$, i.e., $F\left(\Im_{k}\right)=\left\{u \in C: J_{k}(t) u=u, \forall t \in\right.$ $G$. For each $\epsilon>0$ and $p \in G$, we put

$$
F_{\epsilon}\left(J_{k}(p)\right)=\left\{u \in C:\left\|\left(J_{1}(p) u-u, \ldots, J_{k}(p) u-u\right)\right\|_{k} \leq \epsilon\right\} .
$$

Note that if, for any $\epsilon>0$, there exists $p_{\epsilon} \in G$ such that for all $p>p_{\epsilon}, u \in F_{\epsilon}\left(J_{k}(p)\right)$, then $\lim _{p \in G} J_{k}(p) u=u$; moreover, $u \in F\left(\Im_{k}\right)$ by the continuity of elements $\left\{J_{k}(p), p \in G\right\}$ (for more details, we refer to [4-9]).

We denote the set of all almost orbits of $\Im_{k}$ and the set $\left\{J_{k}(p) u_{k}(\cdot): p \in G, u_{k} \in \operatorname{AO}\left(\Im_{k}\right)\right\}$ by $\operatorname{AO}\left(\Im_{k}\right)$ and $\operatorname{LAO}\left(\Im_{k}\right)$, respectively. Denote by $\omega_{\omega}\left(u_{k}\right)$ the set of all weak limit points of subnets of net $\left\{u_{k}(t)\right\}_{t \in G}$.

Lemma 1.6 ([10]) Assume that $X$ is a Banach space and $J$ is the normalized duality function. Therefore

$$
\|u+v\|^{2} \leq\|u\|^{2}+2\langle v, j(u+v)\rangle
$$

for all $j(u+v) \in J(u+v)$ and $u, v \in X$. 
Lemma 1.7 ([11]) Assume that $\left\{\left(X^{k},\|\cdot\|_{k}\right)\right\}_{k \in \mathbb{N}}$ is a uniformly convex multi-Banach space and $\emptyset \neq C \subset X^{k}$ is a bounded closed convex set. Then there exists a strictly increasing continuous convex function $\xi:[0,+\infty) \rightarrow[0,+\infty)$ with $\xi(0)=0$ such that

$$
\begin{aligned}
& \xi\left(\left\|\left(J_{1}\left(\sum_{i=1}^{n} a_{i} u_{i}\right)-\sum_{i=1}^{n} a_{i} J_{1} u_{i}, \ldots, J_{k}\left(\sum_{i=1}^{n} a_{i} u_{i}\right)-\sum_{i=1}^{n} a_{i} J_{k} u_{i}\right)\right\|_{k}\right) \\
& \leq \max _{1 \leq i, j \leq n}\left\{\left\|u_{i}-u_{j}\right\|-\left\|\left(J_{1} u_{i}-J_{1} u_{j}, \ldots, J_{k} u_{i}-J_{k} u_{j}\right)\right\|_{k}\right\}
\end{aligned}
$$

for all integers $a_{1}, \ldots, a_{n} \geq 0, n \geq 1$ with $\sum_{i=1}^{n} a_{i}=1, u_{1}, \ldots, u_{n} \in C$, and every nonexpansive function $J_{k}$ of $C$ to $C$.

Lemma 1.7 implies that, for all $a_{1}, \ldots, a_{n} \geq 0$ with $\sum_{i=1}^{n} a_{i}=1, u_{1}, \ldots, u_{n} \in C$,

$$
\begin{aligned}
& \left\|\left(J_{1}(p)\left(\sum_{i=1}^{n} a_{i} u_{i}\right)-\sum_{i=1}^{n} a_{i} J_{1}(p) u_{i}, \ldots, J_{k}(p)\left(\sum_{i=1}^{n} a_{i} u_{i}\right)-\sum_{i=1}^{n} a_{i} J_{k}(p) u_{i}\right)\right\|_{k} \\
& \leq(1+\alpha(p)) \xi^{-1}\left(\operatorname { m a x } _ { 1 \leq i , j \leq n } \left\{\left\|u_{i}-u_{j}\right\|\right.\right. \\
& \left.\left.\quad-\frac{1}{1+\alpha(p)}\left\|\left(J_{1}(p) u_{i}-J_{1}(p) u_{j}, \ldots, J_{k}(p) u_{i}-J_{k}(p) u_{j}\right)\right\|_{k}\right\}\right) \\
& \leq(1+\alpha(p)) \xi^{-1}\left(\operatorname { m a x } _ { 1 \leq i , j \leq n } \left\{\left\|u_{i}-u_{j}\right\|\right.\right. \\
& \left.\left.\quad-\left\|\left(J_{1}(p) u_{i}-J_{1}(p) u_{j}, \ldots, J_{k}(p) u_{i}-J_{k}(p) u_{j}\right)\right\|_{k}\right\}+d \cdot \alpha(p)\right)
\end{aligned}
$$

in which $d=4 \sup \{\|u\|: u \in C\}+1$.

For every $\epsilon \in(0,1]$, define

$$
a(\epsilon)=\min \left\{\frac{\epsilon^{2}}{(d+2)^{2}}, \frac{\epsilon^{3}}{(3 d+2)^{2}} \xi\left(\frac{\epsilon}{4}\right)\right\}
$$

and

$$
G_{\epsilon}=\{h \in G: \alpha(p) \leq \epsilon\}
$$

in which $\xi(\cdot)$ is as Lemma 1.7. Then $G_{\epsilon} \neq \emptyset$ for $\epsilon>0$, and if $p \in G_{\epsilon}$, then for all $t \geq p, t \in G_{\epsilon}$. Note that $G_{a(\epsilon)} \subset G_{\epsilon}$ for all $\epsilon \in(0,1]$.

\section{Main result}

For studies on ergodic theory and its history, we refer to [4-30]. The results of this paper are an extension and generalization of [31].

Lemma 2.1 For all $p \in G_{a(\epsilon)}$,

$$
\overline{\mathrm{co}} F_{a(\epsilon)}\left(J_{k}(p)\right) \subset F_{\epsilon}\left(J_{K}(p)\right) .
$$


Proof Since $F_{\epsilon}\left(J_{K}(p)\right)$ is closed, we only need to prove that, for all $p \in G_{a(\epsilon)}$,

$$
\operatorname{co} F_{a(\epsilon)}\left(J_{k}(p)\right) \subset F_{\epsilon}\left(J_{K}(p)\right)
$$

Let $v=\sum_{i=1}^{n} a_{i} v_{i}, v_{i} \in F_{a(\epsilon)}\left(J_{k}(p)\right), a_{i} \geq 0, i=1, \ldots, n$, and $\sum_{i=1}^{n} a_{i}=1$. Then

$$
\begin{aligned}
\| & \left(J_{1}(p) v-v, \ldots, J_{k}(p) v-v\right) \|_{k} \\
= & \left\|\left(J_{1}(p) \sum_{i=1}^{n} a_{i} v_{i}-\sum_{i=1}^{n} a_{i} v_{i}, \ldots, J_{k}(p) \sum_{i=1}^{n} a_{i} v_{i}-\sum_{i=1}^{n} a_{i} v_{i}\right)\right\|_{k} \\
\leq & \left\|\left(J_{1}(p) \sum_{i=1}^{n} a_{i} v_{i}-\sum_{i=1}^{n} a_{i} J_{1}(p) v_{i}, \ldots, J_{k}(p) \sum_{i=1}^{n} a_{i} v_{i}-\sum_{i=1}^{n} a_{i} J_{k}(p) v_{i}\right)\right\|_{k} \\
\leq & 2 \xi^{-1}\left(\max _{1 \leq i, j \leq n}\left\{\left\|v_{i}-v_{j}\right\|-\left\|\left(J_{1}(p) v_{i}-J_{1}(p) v_{i}, \ldots, J_{k}(p) v_{i}-J_{k}(p) v_{i}\right)\right\|_{k}\right\}+d \cdot \alpha(p)\right) \\
& +a(\epsilon) \\
\leq & 2 \xi^{-1}\left(\max _{1 \leq i, j \leq n}\left\{\left\|\left(v_{i}-J_{1}(p) v_{i}, \ldots, v_{i}-J_{k}(p) v_{i}\right)\right\|_{k}+\left\|\left(v_{j}-J_{1}(p) v_{j}, \ldots, v_{j}-J_{k}(p) v_{j}\right)\right\|_{k}\right\}\right. \\
& +d \cdot \alpha(p))+a(\epsilon) \\
\leq & 2 \xi^{-1}(2 a(\epsilon)+d \cdot a(\epsilon))+a(\epsilon) \\
\leq & \frac{\epsilon}{2}+\frac{\epsilon}{2}=\epsilon .
\end{aligned}
$$

Lemma 2.2 For every $p \in G_{\frac{\epsilon}{4}}$,

$$
F_{\epsilon}\left(J_{k}(p)\right)+B\left(0, \frac{\epsilon}{4}\right) \subset F_{\epsilon}\left(J_{k}(p)\right)
$$

Proof Let $p \in G_{\frac{\epsilon}{4}}$ and $u=v+w \in F_{\frac{\epsilon}{4}}\left(J_{k}(p)\right)+B\left(0, \frac{\epsilon}{4}\right)$, where $v \in F_{\frac{\epsilon}{4}}\left(J_{k}(p)\right)$ and $w \in B\left(0, \frac{\epsilon}{4}\right)$, then

$$
\begin{aligned}
\|( & \left.J_{1}(p) u-u, \ldots, J_{k}(p) u-u\right) \|_{k} \\
= & \left\|\left(J_{1}(p)(v+w)-(v+w), \ldots, J_{k}(p)(v+w)-(v+w)\right)\right\|_{k} \\
\leq & \left\|\left(J_{1}(p)(v+w)-J_{1}(p) v, \ldots, J_{k}(p)(v+w)-J_{k}(p) v\right)\right\|_{k} \\
& \quad+\left\|\left(J_{1}(p) v-v, \ldots, J_{k}(p) v-v\right)\right\|_{k}+\|w\| \\
\leq & 2\|w\|+\left\|\left(J_{1}(p) v-v, \ldots, J_{k}(p) v-v\right)\right\|_{k}+\|w\| \\
\leq & 3 \frac{\epsilon}{4}+\frac{\epsilon}{4}=\epsilon .
\end{aligned}
$$

Lemma 2.3 Assume that $\epsilon \in(0,1]$ and $p \in G_{a\left(a\left(\frac{\epsilon}{4}\right)\right)}$, so we can find $n_{0} \in N$ such that, for each $n \geq n_{0}$ and $u \in C$,

$$
\frac{1}{n} \sum_{i=1}^{n} J_{k}\left(p^{i}\right) u \in F_{\epsilon}\left(J_{k}(p)\right) .
$$


Proof Let $\epsilon \in(0,1]$ and $m=\frac{2 d+1}{a\left(\frac{\epsilon}{4}\right)}$. There is $n_{0} \in N$ satisfying

$$
n_{0} \geq \max \left\{\frac{12 m d}{\epsilon}, 32 m^{2} d(d+1)\left(\xi\left(\frac{a\left(\frac{\epsilon}{4}\right)}{2}\right) \epsilon\right)^{-1}\right\}
$$

For any $n \geq n_{0}$ and $p \in G_{a\left(a\left(\frac{\epsilon}{4}\right)\right)}$, we can take a number

$$
K=m^{2} d(1+2 n \alpha(p))\left(\xi\left(\frac{a\left(\frac{\epsilon}{4}\right)}{2}\right)\right)^{-1} \quad\left(k<\frac{n}{2}\right) .
$$

For every $i \in N$ and $u \in C$, we put

$$
\begin{aligned}
a_{i}(u)= & \xi\left(\frac{8}{9} \|\left(\frac{1}{m} \sum_{j=1}^{m} J_{1}\left(p^{i+j+1}\right) u-J_{1}(p) \frac{1}{m} \sum_{j=1}^{m} J_{1}\left(p^{i+j}\right) u, \ldots,\right.\right. \\
& \left.\left.\frac{1}{m} \sum_{j=1}^{m} J_{k}\left(p^{i+j+1}\right) u-J_{k}(p) \frac{1}{m} \sum_{j=1}^{m} J_{k}\left(p^{i+j}\right) u\right) \|_{k}\right) .
\end{aligned}
$$

By $\alpha(p) \leq \frac{1}{8}$ and

$$
\begin{aligned}
a_{i}(u) \leq & \max _{1 \leq j, t \leq m}\left\{\left\|J_{1}\left(p^{i+j}\right) u-J_{k}\left(p^{i+t}\right) u, \ldots, J_{k}\left(p^{i+j}\right) u-J_{k}\left(p^{i+t}\right) u\right\|_{k}\right. \\
& \left.-\left\|J_{1}\left(p^{i+j+1}\right) u-J_{k}\left(p^{i+t+1}\right) u, \ldots, J_{k}\left(p^{i+j+1}\right) u-J_{k}\left(p^{i+t+1}\right) u\right\|_{k}+d \cdot \alpha(p)\right\} \\
\leq & \sum_{1 \leq j<t \leq m}\left(\left\|\left(J_{1}\left(p^{i+j}\right) u-J_{1}\left(p^{i+t}\right) u, \ldots J_{k}\left(p^{i+j}\right) u-J_{k}\left(p^{i+t}\right) u\right)\right\|_{k}\right. \\
& \left.-\left\|\left(J_{1}\left(p^{i+j+1}\right) u-J_{1}\left(p^{i+t+1}\right) u, \ldots J_{k}\left(p^{i+j+1}\right) u-J_{k}\left(p^{i+t+1}\right) u\right)\right\|_{k}+d \alpha(p)\right),
\end{aligned}
$$

we get

$$
\begin{aligned}
& \sum_{i=1}^{n} a_{i}(u) \\
& \quad \leq \sum_{i=1}^{n} \sum_{1 \leq j<t \leq m}\left(\left\|J_{1}\left(p^{i+j}\right) u-J_{k}\left(p^{i+t}\right) u, \ldots, J_{k}\left(p^{i+j}\right) u-J_{k}\left(p^{i+t}\right) u\right\|_{k}\right. \\
& \left.\quad-\left\|J_{1}\left(p^{i+j+1}\right) u-J_{k}\left(p^{i+t+1}\right) u, \ldots, J_{k}\left(p^{i+j+1}\right) u-J_{k}\left(p^{i+t+1}\right) u\right\|_{k}+d \cdot \alpha(p)\right) \\
& =\sum_{1 \leq j<t \leq m} \sum_{i=1}^{n}\left(\left\|J_{1}\left(p^{i+j}\right) u-J_{k}\left(p^{i+t}\right) u, \ldots, J_{k}\left(p^{i+j}\right) u-J_{k}\left(p^{i+t}\right) u\right\|_{k}\right. \\
& \left.\quad-\left\|J_{1}\left(p^{i+j+1}\right) u-J_{k}\left(p^{i+t+1}\right) u, \ldots, J_{k}\left(p^{i+j+1}\right) u-J_{k}\left(p^{i+t+1}\right) u\right\|_{k}+d \cdot \alpha(p)\right) \\
& \leq \sum_{1 \leq j<t \leq m}(d+n d \cdot \alpha(p)) \leq m^{2} d(1+n \alpha(p)) .
\end{aligned}
$$

Suppose that there is an element say $t$ in $\left\{a_{i}(u): i=1,2, \ldots, 2 n\right\}$ such that if $a_{i}(u) \geq \xi\left(\frac{a\left(\frac{\epsilon}{4}\right)}{2}\right)$, then

$$
t \xi\left(\frac{a\left(\frac{\epsilon}{4}\right)}{2}\right) \leq m^{2} d(1+2 n \alpha(p))
$$


Hence

$$
t \leq m^{2} d(1+2 n \alpha(p))\left(\xi\left(\frac{a\left(\frac{\epsilon}{4}\right)}{2}\right)\right)^{-1}=K .
$$

So, there are at most $N=[K]$ terms in $\left\{a_{i}(u): i=1,2, \ldots, 2 n\right\}$ with $a_{i}(u) \geq \xi\left(\frac{a\left(\frac{\epsilon}{4}\right)}{2}\right)$. Then, for every $i$ in $\{1,2, \ldots, n\}$, there exists at least one term $a_{i+j_{0}}(u)\left(0 \leq j_{0} \leq N\right)$ in $\left\{a_{i+j}(u): j=\right.$ $0,1, \ldots, N\}$ hold $a_{i+j_{0}}<\xi\left(\frac{a\left(\frac{\epsilon}{4}\right)}{2}\right)$.

Put

$$
\ell_{i}=\min \left\{j: a_{i+j}(u)<\xi\left(\frac{a\left(\frac{\epsilon}{4}\right)}{2}\right), 0 \leq j \leq N\right\},
$$

$i=1,2, \ldots, n$. Now, there are at most $N$ elements in $\{i=1,2, \ldots, n\}$ such that $\ell_{i} \neq 0$. Since

$$
\begin{aligned}
& \|\left(J_{1}(p) \frac{1}{m} \sum_{j=1}^{m} J_{1}\left(p^{i+\ell_{i}+j}\right) u-\frac{1}{m} \sum_{j=1}^{m} J_{1}\left(p^{i+\ell_{i}+j}\right) u, \ldots,\right. \\
& \left.J_{k}(p) \frac{1}{m} \sum_{j=1}^{m} J_{k}\left(p^{i+\ell_{i}+j}\right) u-\frac{1}{m} \sum_{j=1}^{m} J_{k}\left(p^{i+\ell_{i}+j}\right) u\right) \|_{k} \\
& \leq \|\left(J_{1}(p) \frac{1}{m} \sum_{j=1}^{m} J_{1}\left(p^{i+\ell_{i}+j}\right) u-\frac{1}{m} \sum_{j=1}^{m} J_{1}\left(p^{i+\ell_{i}+j+1}\right) u, \ldots,\right. \\
& \left.\quad J_{k}(p) \frac{1}{m} \sum_{j=1}^{m} J_{k}\left(p^{i+\ell_{i}+j}\right) u-\frac{1}{m} \sum_{j=1}^{m} J_{k}\left(h^{i+\ell_{i}+j+1}\right) u\right) \|_{k} \\
& \quad \|\left(\frac{1}{m} \sum_{j=1}^{m} J_{1}\left(p^{i+\ell_{i}+j}\right) u-\frac{1}{m} \sum_{j=1}^{m} J_{1}\left(p^{i+\ell_{i}+j+1}\right) u, \ldots,\right. \\
& \left.\leq \frac{1}{m} \sum_{j=1}^{m} J_{k}\left(p^{i+\ell_{i}+j}\right) u-\frac{1}{m} \sum_{j=1}^{m} J_{k}\left(p^{i+\ell_{i}+j+1}\right) u\right) \|_{k} \\
& \left.\leq \frac{9}{8} \xi^{-1}\left(a_{i+\ell_{i}}(u)\right)+\frac{\epsilon}{2 m}\right)+\frac{1}{4} a\left(\frac{\epsilon}{4}\right)<a\left(\frac{\epsilon}{4}\right), \\
& \frac{9}{4}\left(\frac{\epsilon}{4}\right)
\end{aligned}
$$

we can conclude that, for all $p \in G_{a\left(a\left(\frac{\epsilon}{4}\right)\right)}$,

$$
\frac{1}{m} \sum_{j=1}^{m} J_{k}\left(p^{i+\ell_{i}+j}\right) u \in F_{a\left(\frac{\epsilon}{4}\right)}\left(J_{k}(p)\right)
$$

By Lemma 2.1, we get, for all $p \in G_{a\left(a\left(\frac{\epsilon}{4}\right)\right)} \subset G_{a\left(\frac{\epsilon}{4}\right)}$,

$$
\frac{1}{n} \sum_{i=1}^{n} \frac{1}{m} \sum_{j=1}^{m} J_{k}\left(p^{i+\ell_{i}+j}\right) u \in \operatorname{co} F_{a\left(\frac{\epsilon}{4}\right)}\left(J_{k}(p)\right) \subset F_{\frac{\epsilon}{4}}\left(J_{k}(p)\right) .
$$


Using Lemma 2.2 and

$$
\begin{aligned}
\| & \left(\frac{1}{n} \sum_{i=1}^{n} J_{1}\left(p^{i}\right) u-\frac{1}{n} \sum_{i=1}^{n} \frac{1}{m} \sum_{j=1}^{m} J_{1}\left(p^{i+\ell_{i}+j}\right) u, \ldots,\right. \\
& \left.\frac{1}{n} \sum_{i=1}^{n} J_{k}\left(p^{i}\right) u-\frac{1}{n} \sum_{i=1}^{n} \frac{1}{m} \sum_{j=1}^{m} J_{k}\left(p^{i+\ell_{i}+j}\right) u\right) \|_{k} \\
\leq & \frac{1}{m n} \sum_{j=1}^{m}\left\|\left(\sum_{i=1}^{n} J_{1}\left(p^{i}\right) u-\sum_{i=1}^{n} J_{1}\left(p^{i+\ell_{i}+j}\right) u, \ldots, \sum_{i=1}^{n} J_{k}\left(p^{i}\right) u-s u m_{i=1}^{n} J_{k}\left(p^{i+\ell_{i}+j}\right) u\right)\right\|_{k} \\
\leq & \frac{1}{m n} \sum_{j=1}^{m}\left\|\left(\sum_{i=1}^{n} J_{1}\left(p^{i}\right) u-\sum_{i=1}^{n} J_{1}\left(p^{i+j}\right) u, \ldots, \sum_{i=1}^{n} J_{k}\left(p^{i}\right) u-\sum_{i=1}^{n} J_{k}\left(p^{i+j}\right) u\right)\right\|_{k} \\
& +\frac{1}{m n} \sum_{j=1}^{m} \|\left(\sum_{i=1}^{n} L_{1}\left(p^{i+j}\right) u-\sum_{i=1}^{n} J_{1}\left(p^{i+\ell_{i}+j}\right) u, \ldots,\right. \\
\leq & \frac{\epsilon}{12}+\frac{\epsilon}{32}+\frac{\epsilon}{8}<\frac{\epsilon}{4}, \\
\leq & \frac{\epsilon}{n}+\frac{m^{2} d^{2}\left(\xi\left(\frac{a\left(\frac{\epsilon}{4}\right)}{2}\right)\right)^{-1}}{n}+2 m^{2} d^{2} \alpha(p)\left(\xi\left(\frac{a\left(\frac{\epsilon}{4}\right)}{2}\right)\right)^{-1} \\
\leq & N d \\
\leq &
\end{aligned}
$$

we obtain

$$
\frac{1}{n} \sum_{i=1}^{n} J_{k}\left(p^{i}\right) u \in F_{\frac{\epsilon}{4}}\left(J_{k}(p)\right)+B\left(0, \frac{\epsilon}{4}\right) \subset F_{\epsilon}\left(J_{k}(p)\right)
$$

Lemma 2.4 Suppose that $u_{k}(\cdot)$ is an almost orbit of $\Im_{k}$. So

$$
\lim _{t \in G}\left\|\left(\gamma u_{1}(t)+(1-\gamma) \varphi-g, \ldots, \gamma u_{k}(t)+(1-\gamma) \varphi-g\right)\right\|_{k}
$$

exist for every $\gamma \in(0,1)$ and $\varphi, g \in F\left(\Im_{k}\right)$.

Proof To complete the proof, it is enough to prove that

$$
\begin{aligned}
& \inf _{s \in G} \sup _{t \in G}\left\|\left(\gamma u_{1}(t s)+(1-\gamma) \varphi-g, \ldots, \gamma u_{k}(t s)+(1-\gamma) \varphi-g\right)\right\|_{k} \\
& \quad \leq \sup _{s \in G} \inf _{t \in G}\left\|\left(\gamma u_{1}(t s)+(1-\gamma) \varphi-g, \ldots, \gamma u_{k}(t s)+(1-\gamma) \varphi-g\right)\right\|_{k} .
\end{aligned}
$$

We know, for every $\epsilon>0$, there are $t_{0}$ and $s_{0} \in G$ such that, for any $t \in G, \alpha\left(t t_{0}\right)<\frac{\epsilon}{1+d}$ and $\varphi\left(t s_{0}\right)<\epsilon$, where $\varphi(t)=\sup _{p \in G}\left\|\left(u_{1}(p t)-J_{1}(p) u_{1}(t), \ldots, u_{k}(p t)-J_{k}(p) u_{k}(t)\right)\right\|_{k}$. So, for every 
$a \in G$,

$$
\begin{aligned}
& \inf _{s \in G} \sup _{t \in G}\left\|\left(u_{1}\left(t s s_{0}\right)-\varphi, \ldots, u_{k}\left(t s s_{0}\right)-\varphi\right)\right\|_{k} \\
& \leq \sup _{t \in G}\left\|\left(u_{1}\left(t t_{0} a s_{0}\right)-\varphi, \ldots, u_{k}\left(t t_{0} a s_{0}\right)-\varphi\right)\right\|_{k} \\
& \leq \sup _{t \in G}\left\|\left(u_{1}\left(t t_{0} a s_{0}\right)-J_{1}\left(t t_{0}\right) u_{1}\left(a s_{0}\right), \ldots, u_{k}\left(t t_{0} a s_{0}\right)-J_{k}\left(t t_{0}\right) u_{k}\left(a s_{0}\right)\right)\right\|_{k} \\
& \quad \quad+\sup _{t \in G}\left\|J_{1}\left(t t_{0}\right) u_{1}\left(a s_{0}\right)-\varphi, \ldots, J_{k}\left(t t_{0}\right) u_{k}\left(a s_{0}\right)-\varphi\right\|_{k} \\
& \leq \varphi\left(a s_{0}\right)+\sup _{t \in G}\left(1+\alpha\left(t t_{0}\right)\right) \cdot\left\|\left(u_{1}\left(a s_{0}\right)-\varphi, \ldots, u_{k}\left(a s_{0}\right)-\varphi\right)\right\|_{k} \\
& \leq\left\|\left(u_{1}\left(a s_{0}\right)-\varphi, \ldots, u_{k}\left(a s_{0}\right)-\varphi\right)\right\|_{k}+2 \epsilon .
\end{aligned}
$$

Hence

$$
\inf _{s \in G} \sup _{t \in G}\left\|\left(u_{1}\left(t s s_{0}\right)-\varphi, \ldots, u_{k}\left(t s s_{0}\right)-\varphi\right)\right\|_{k} \leq \inf _{a \in G}\left\|\left(u_{1}\left(a s_{0}\right)-\varphi, \ldots, u_{k}\left(a s_{0}\right)-\varphi\right)\right\|_{k}+2 \epsilon
$$

Thus, there exists $s_{1} \in G$ such that

$$
\sup _{t \in G}\left\|\left(u_{1}\left(t s_{1} s_{0}\right)-\varphi, \ldots, u_{k}\left(t s_{1} s_{0}\right)-\varphi\right)\right\|_{k}<\inf _{a \in G}\left\|\left(u_{1}\left(a s_{0}\right)-\varphi, \ldots, u_{k}\left(a s_{0}\right)-\varphi\right)\right\|_{k}+3 \epsilon .
$$

Then, for every $a \in G$, we get

$$
\begin{aligned}
\inf _{s \in G} \sup _{t \in G}\left\|\left(\gamma u_{1}(t s)+(1-\gamma) \varphi-g, \ldots, \gamma u_{k}(t s)+(1-\gamma) \varphi-g\right)\right\|_{k} \\
\leq \sup _{t \in G}\left\|\left(\gamma u_{1}\left(t t_{0} a s_{1} s_{0}\right)+(1-\gamma) \varphi-g, \ldots, \gamma u_{k}\left(t t_{0} a s_{1} s_{0}\right)+(1-\gamma) \varphi-g\right)\right\|_{k} \\
\leq \gamma \sup _{t \in G}\left\|\left(u_{1}\left(t t_{0} a s_{1} s_{0}\right)-J_{1}\left(t t_{0}\right) u_{1}\left(a s_{1} s_{0}\right), \ldots, u_{k}\left(t t_{0} a s_{1} s_{0}\right)-J_{k}\left(t t_{0}\right) u_{k}\left(a s_{1} s_{0}\right)\right)\right\|_{k} \\
\quad+\sup _{t \in G}\left\|\left(\gamma J_{1}\left(t t_{0}\right) u_{1}\left(a s_{1} s_{0}\right)+(1-\gamma) \varphi-g, \ldots, \gamma J_{k}\left(t t_{0}\right) u_{k}\left(a s_{1} s_{0}\right)+(1-\gamma) \varphi-g\right)\right\|_{k} \\
\leq \quad \varphi\left(a s_{1} s_{0}\right)+\sup _{t \in G} \|\left(\gamma J_{1}\left(t t_{0}\right) u_{1}\left(a s_{1} s_{0}\right)+(1-\gamma) \varphi-J_{1}\left(t t_{0}\right)\left(\gamma u_{1}\left(a s_{1} s_{0}\right)+(1-\gamma) \varphi\right), \ldots\right. \\
\left.\quad \gamma J_{k}\left(t t_{0}\right) u_{k}\left(a s_{1} s_{0}\right)+(1-\gamma) \varphi-J_{k}\left(t t_{0}\right)\left(\gamma u_{k}\left(a s_{1} s_{0}\right)+(1-\gamma) \varphi\right)\right) \|_{k} \\
\quad+\sup _{t \in G} \|\left(J_{1}\left(t t_{0}\right)\left(\gamma u_{1}\left(a s_{1} s_{0}\right)+(1-\gamma) \varphi\right)-g, \ldots,\right. \\
\left.\quad J_{k}\left(t t_{0}\right)\left(\gamma u_{k}\left(a s_{1} s_{0}\right)+(1-\gamma) \varphi\right)-g\right) \|_{k} \\
\quad+\epsilon \sup _{t \in G}\left(1+\alpha\left(t t_{0}\right)\right) \xi^{-1}\left(\left\|\left(u_{1}\left(a s_{1} s_{0}\right)-\varphi, \ldots, u_{k}\left(a s_{1} s_{0}\right)-\varphi\right)\right\|_{k}\right. \\
\left.\quad-\left\|\left(J_{1}\left(t t_{0}\right) u_{1}\left(a s_{1} s_{0}\right)-\varphi, \ldots, J_{k}\left(t t_{0}\right) u_{k}\left(a s_{1} s_{0}\right)-\varphi\right)\right\|_{k}+d \cdot \alpha\left(t t_{0}\right)\right) \\
\quad+\sup _{t \in G}\left(1+\alpha\left(t t_{0}\right)\right)\left\|\left(\gamma u_{1}\left(a s_{1} s_{0}\right)+(1-\gamma) \varphi-g, \ldots, \gamma u_{k}\left(a s_{1} s_{0}\right)+(1-\gamma) \varphi-g\right)\right\|_{k} \\
\leq \epsilon+(1-\epsilon) \sup _{t \in G} \xi^{-1}\left(\left\|\left(u_{1}\left(a s_{1} s_{0}\right)-\varphi, \ldots, u_{k}\left(a s_{1} s_{0}\right)-\varphi\right)\right\|_{k}\right. \\
\left.\quad-\left\|\left(u_{1}\left(t t_{0} a s_{1} s_{0}\right)-\varphi, \ldots, u_{k}\left(t t_{0} a s_{1} s_{0}\right)-\varphi\right)\right\|_{k}+\varphi\left(a s_{1} s_{0}\right)+\epsilon\right)
\end{aligned}
$$




$$
\begin{aligned}
& +(1+\epsilon)\left\|\left(\gamma u_{1}\left(a s_{1} s_{0}\right)+(1-\gamma) \varphi-g, \ldots, \gamma u_{k}\left(a s_{1} s_{0}\right)+(1-\gamma) \varphi-g\right)\right\|_{k} \\
\leq & +(1+\epsilon) \xi^{-1}(5 \epsilon) \\
& +(1+\epsilon)\left\|\left(\gamma u_{1}\left(a s_{1} s_{0}\right)+(1-\gamma) \varphi-g, \ldots, \gamma u_{k}\left(a s_{1} s_{0}\right)+(1-\gamma) \varphi-g\right)\right\|_{k} .
\end{aligned}
$$

Then

$$
\begin{aligned}
& \inf _{s \in G} \sup _{t \in G}\left\|\left(\gamma u_{1}(t s)+(1-\gamma) \varphi-g, \ldots, \gamma u_{k}(t s)+(1-\gamma) \varphi-g\right)\right\|_{k} \\
& \leq \epsilon(1+\epsilon) \xi^{-1}(5 \epsilon) \\
&+(1+\epsilon) \inf _{a \in G}\left\|\left(\gamma u_{1}\left(a s_{1} s_{0}\right)+(1-\gamma) \varphi-g, \ldots, \gamma u_{k}\left(a s_{1} s_{0}\right)+(1-\gamma) \varphi-g\right)\right\|_{k} \\
& \leq \epsilon(1+\epsilon) \xi^{-1}(5 \epsilon) \\
&+(1+\epsilon) \sup _{b \in G} \inf _{a \in G}\left\|\left(\gamma u_{1}(a b)+(1-\gamma) \varphi-g, \ldots, \gamma u_{k}(a b)+(1-\gamma) \varphi-g\right)\right\|_{k} .
\end{aligned}
$$

Hence,

$$
\begin{aligned}
& \inf _{s \in G} \sup _{t \in G}\left\|\left(\gamma u_{1}(t s)+(1-\gamma) \varphi-g, \ldots, \gamma u_{k}(t s)+(1-\gamma) \varphi-g\right)\right\|_{k} \\
& \quad \leq \sup _{s \in G} \inf _{t \in G}\left\|\left(\gamma u_{1}(t s)+(1-\gamma) \varphi-g, \ldots, \gamma u_{k}(t s)+(1-\gamma) \varphi-g\right)\right\|_{k}
\end{aligned}
$$

because $\epsilon>0$ is arbitrary.

Theorem 2.5 Assume that $\left\{\left(X^{k},\|\cdot\|_{k}\right)\right\}_{k \in \mathbb{N}}$ is a uniformly convex multi-Banach space, and suppose that $\emptyset \neq C \subset X$ is bounded and closed. Assume that $\Im_{k}=\left\{J_{k}(t): t \in G\right\}$ for each $k \geq 1$ is a reversible semigroup of asymptotically nonexpansive functions on $C$. If $D$ has a left invariant mean, then there exists a retraction $P_{k}$ from $\mathrm{LAO}(\Im)$ onto $F\left(\Im_{k}\right)$ in which:

(1) $P_{k}$ is nonexpansive in the sense

$$
\begin{aligned}
& \left\|\left(P_{1} u_{1}-P_{1} v_{1}, \ldots, P_{k} u_{k}-P_{k} v_{k}\right)\right\|_{k} \\
& \quad \leq \inf _{s \in G} \sup _{t \in G}\left\|\left(u_{1}(s t)-v_{1}(s t), \ldots, u_{k}(s t)-v_{k}(s t)\right)\right\|_{k^{\prime}}, \quad \forall u_{k}, v_{k} \in \operatorname{LAO}\left(\Im_{k}\right) ;
\end{aligned}
$$

(2) $P_{k} J_{k}(p) u_{k}=J_{k}(p) P_{k} u_{k}=P_{k} u_{k}$ for all $u_{k} \in \mathrm{AO}\left(\Im_{k}\right)$ and $p \in G$;

(3) $P_{k} u_{k} \in \bigcap_{s \in G} \overline{\operatorname{conv}}\left\{u_{k}(t): t \geq s\right\}$ for all $u_{k} \in \operatorname{LAO}\left(\Im_{k}\right)$.

Proof We know $D$ has a left invariant mean, so there is a net $\left\{\gamma_{k, \alpha}: \alpha \in A\right\}$ of finite means on $G$ in which $\lim _{\alpha \in A}\left\|\left(\gamma_{1, \alpha}-\ell_{s}^{*} \gamma_{1, \alpha}, \ldots, \gamma_{k, \alpha}-\ell_{s}^{*} \gamma_{k, \alpha}\right)\right\|_{k}=0$ for every $s \in G$, in which $A$ is a directed system. Putting $I=A \times G=\{\beta=(\alpha, t): \alpha \in A, t \in G\}$. For $\beta_{i}=\left(\alpha_{i}, t_{i}\right) \in I, i=1,2$, define $\beta_{1} \leq \beta_{2}$ iff $\alpha_{1} \leq \alpha_{2}, t_{1} \leq t_{2}$. Then, $I$ is also a directed system. For each $\beta=(\alpha, t) \in I$, define $P_{k, 1} \beta=\alpha, P_{k, 2} \beta=t$, and $\gamma_{\beta}=\gamma_{\alpha}$. So, for every $s \in G$,

$$
\lim _{\beta \in I}\left\|\left(\gamma_{1, \beta}-\ell^{*} \gamma_{1, \beta}, \ldots, \gamma_{k, \beta}-\ell^{*} \gamma_{k, \beta}\right)\right\|_{k}=0
$$

Assume that $\gamma=\left\{\left\{t_{\beta}\right\}_{\beta \in I}, t_{\beta} \geq P_{k, 2} \beta, \forall \beta \in I\right\}$. Taking any $\left\{t_{\beta}, \beta \in I\right\} \in \gamma$, since $r_{t \beta}^{*} \gamma_{k, \beta}$ is bounded, without loss of generality, let $r_{t \beta}^{*} \gamma_{k, \beta}$ be weak* convergent. Then, for all $u_{k} \in$ 
$\operatorname{LAO}\left(\Im_{k}\right), \omega-\lim _{\beta \in I} \gamma_{k, \beta}(t)\left\langle u_{k}\left(t t_{\beta}\right)\right\rangle$ exist. We define

$$
P_{k} u_{k}=\omega-\lim _{\beta \in I} \gamma_{k, \beta}(t)\left\langle u_{k}\left(t t_{\beta}\right)\right\rangle
$$

On the other hand, for every $u_{k} \in \mathrm{LAO}\left(\Im_{k}\right), P_{k} u_{k} \in \bigcap_{s \in G} \overline{\operatorname{conv}}\{u(t): t \geq s\}$. Next, we shall show that $P_{k} u_{k} \in F\left(\Im_{k}\right)$. Then, for every $\epsilon \in(0,1]$, there is $t_{0} \in G$ such that, for each $t \geq t_{0}$, $\varphi(t)<\frac{a(\epsilon)}{4}$. Also, we can suppose that $P_{k 2} \beta \geq t_{0}$ for every $\beta \in I$, so $t_{\beta} \geq t_{0},\left\{t_{\beta}\right\} \in \gamma$. From Lemma 2.3, for every $p \in G_{a\left(a\left(\frac{a(\epsilon)}{16}\right)\right)}$, there is $n \in N$ such that, for each $t \in G$ and $\beta \in I$,

$$
\frac{1}{n} \sum_{i=1}^{n} J_{k}\left(p^{i}\right) u_{k}\left(t t_{\beta}\right) \in F_{\frac{a(\epsilon)}{4}}\left(J_{k}(p)\right) .
$$

Since for every $t \in G$

$$
\begin{aligned}
& \left\|\left(\frac{1}{n} \sum_{i=1}^{n} J_{1}\left(p^{i}\right) u_{1}\left(t t_{\beta}\right)-\frac{1}{n} \sum_{i=1}^{n} u_{1}\left(p^{i} t t_{\beta}\right), \ldots, \frac{1}{n} \sum_{i=1}^{n} J_{k}\left(p^{i}\right) u_{k}\left(t t_{\beta}\right)-\frac{1}{n} \sum_{i=1}^{n} u_{k}\left(p^{i} t t_{\beta}\right)\right)\right\|_{k} \\
& \quad \leq \varphi\left(t t_{\beta}\right)<\frac{a(\epsilon)}{4}
\end{aligned}
$$

we have, for every $p \in G_{a\left(a\left(\frac{a(\epsilon)}{16}\right)\right)}$,

$$
\frac{1}{n} \sum_{i=1}^{n} u_{k}\left(p^{i} t t_{\beta}\right) \in F_{\frac{a(\epsilon)}{4}}\left(J_{k}(p)\right)+B\left(0, \frac{a(\epsilon)}{4}\right) \subset F_{a(\epsilon)}\left(J_{k}(p)\right) .
$$

Equation (2.1) implies that

$$
\begin{gathered}
\lim _{\beta \in I} \|\left\langle\gamma_{1, \beta}(t)\left\langle\frac{1}{n} \sum_{i=1}^{n} u_{1}\left(p^{i} t t_{\beta}\right)\right\rangle-\gamma_{1, \beta}\left\langle u_{1}\left(t t_{\beta}\right)\right\rangle, \ldots,\right. \\
\left.\gamma_{k, \beta}(t)\left\langle\frac{1}{n} \sum_{i=1}^{n} u_{k}\left(p^{i} t t_{\beta}\right)\right\rangle-\gamma_{k, \beta}\left\langle u_{k}\left(t t_{\beta}\right)\right\rangle\right) \|_{k}=0 .
\end{gathered}
$$

Combining it with the definition of $P_{k} u_{k}$, we get, for all $p \in G_{a\left(a\left(\frac{a(\epsilon)}{16}\right)\right)}$ '

$$
P_{k} u_{k}=\omega-\lim _{\beta \in I} \gamma_{k, \beta}(t)\left\langle\frac{1}{n} \sum_{i=1}^{n} u_{k}\left(p^{i} t t_{\beta}\right)\right\rangle \in \overline{\operatorname{co}} F_{a(\epsilon)}\left(J_{k}(p)\right) .
$$

Lemma 2.1 also implies that for every $p \in G_{a\left(a\left(\frac{a(\epsilon)}{16}\right)\right)}, P_{k} u_{k} \in F_{\epsilon}\left(J_{k}(p)\right)$. Now, the continuity of $J_{k}(p)$ implies that $P_{k} u_{k} \in F\left(\Im_{k}\right)$. Obviously, for any $p \in G$,

$$
\begin{aligned}
P_{k} J_{k}(p) u_{k} & =\omega-\lim _{\beta \in I} \gamma_{k, \beta}(t)\left\langle J_{k}(p) u_{k}\left(t t_{\beta}\right)\right\rangle \\
& =\omega-\lim _{\beta \in I} \gamma_{k, \beta}(t)\left\langle u_{k}\left(h t t_{\beta}\right)\right\rangle \\
& =\omega-\lim _{\beta \in I} \gamma_{k, \beta}(t)\left\langle u_{k}\left(t t_{\beta}\right)\right\rangle \quad \text { (using (2.1)) } \\
& =P_{k} u_{k}
\end{aligned}
$$


and for every $v_{k} \in \operatorname{LAO}\left(\Im_{k}\right)$ and $s \in G$, we have

$$
\begin{aligned}
\| & \left(P_{1} u_{1}-P_{1} v_{1}, \ldots, P_{1} u_{1}-P_{1} v_{1}\right) \|_{k} \\
\leq & \liminf _{\beta \in I}\left\|\left(\gamma_{1, \beta}(t)\left\langle u_{1}\left(t t_{\beta}\right)\right\rangle-\gamma_{1, \beta}(t)\left\langle v_{1}\left(t t_{\beta}\right)\right\rangle, \ldots, \gamma_{k, \beta}(t)\left\langle u_{k}\left(t t_{\beta}\right)\right\rangle-\gamma_{k, \beta}(t)\left\langle v_{k}\left(t t_{\beta}\right)\right\rangle\right)\right\|_{k} \\
= & \liminf _{\beta \in I} \|\left(\gamma_{1, \beta}(t)\left\langle u_{1}\left(s t t_{\beta}\right)\right\rangle-\gamma_{1, \beta}(t)\left\langle v_{1}\left(s t t_{\beta}\right)\right\rangle, \ldots,\right. \\
& \left.\gamma_{k, \beta}(t)\left\langle u_{k}\left(s t t_{\beta}\right)\right\rangle-\gamma_{k, \beta}(t)\left\langle v_{k}\left(s t t_{\beta}\right)\right\rangle\right) \|_{k}(\text { by }(2.1)) \\
\leq & \liminf _{\beta \in I}\left\|\left(\gamma_{1, \beta}(t), \ldots, \gamma_{1, \beta}(t)\right)\right\|_{k} \cdot \sup _{t \in G}\left\|\left(u_{1}\left(s t t_{\beta}\right)-v_{1}\left(s t t_{\beta}\right), \ldots, u_{k}\left(s t t_{\beta}\right)-v_{k}\left(s t t_{\beta}\right)\right)\right\|_{k} \\
\leq & \sup _{t \in G}\left\|\left(u_{1}(s t)-v_{1}(s t), \ldots, u_{k}(s t)-v_{k}(s t)\right)\right\|_{k} .
\end{aligned}
$$

Thus,

$$
\left\|\left(P_{1} u_{1}-P_{1} v_{1}, \ldots, P_{k} u_{k}-P_{k} v_{k}\right)\right\|_{k} \leq \inf _{s \in G} \sup _{t \in G}\left\|\left(u_{1}(s t)-v_{1}(s t), \ldots, u_{k}(s t)-v_{k}(s t)\right)\right\|_{k} .
$$

Theorem 2.6 (Ergodic theorem [17]) Assume that $\left\{\left(X^{k},\|\cdot\|_{k}\right)\right\}_{k \in \mathbb{N}}$ is a uniformly convex multi-Banach space, and suppose that $\emptyset \neq C \subset X$ is bounded and closed. Assume that $\Im_{k}=$ $\left\{J_{k}(t): t \in G\right\}$ is a reversible semigroup of asymptotically nonexpansive functions on $C$. If $D$ has a left invariant mean and there is a unique retraction $P_{k}$ from $\operatorname{LAO}\left(\Im_{K}\right)$ onto $F\left(\Im_{k}\right)$, which satisfies properties (1)-(3) in Theorem 2.5, then for every strongly net $\left\{v_{k, \alpha}: \alpha \in A\right\}$ on $D$ and $u_{k} \in \mathrm{AO}\left(\Im_{k}\right)$,

$$
\omega-\lim _{\alpha \in A} \int u_{k}(t p) d v_{k, \alpha}(t)=P_{k} \in F\left(\Im_{k}\right) \quad \text { uniformly in } p \in \gamma(G)
$$

in which $\gamma(G)=\{s \in G: s t=$ ts for all $t \in G\}$.

Theorem 2.7 Assume that $\left\{\left(X^{k},\|\cdot\|_{k}\right)\right\}_{k \in \mathbb{N}}$ is a uniformly convex multi-Banach space, and suppose that $\emptyset \neq C \subset X$ is bounded and closed. Assume that $\Im_{k}=\left\{J_{k}(t): t \in G\right\}$ of a reversible semigroup of asymptotically nonexpansive mappings on $C$, and let $u_{k}(\cdot)$ be an almost orbit of $\Im_{k}$. If

$$
\omega-\lim _{t \in G} u_{k}(p t)-u_{k}(t)=0
$$

for every $p \in G$, then

$$
\omega_{\omega}\left(u_{k}\right) \subset F\left(\Im_{k}\right) .
$$

Proof Let $\epsilon \in(0,1]$, then there is $t_{0} \in G$ such that, for $t \geq t_{0}, \varphi(t)<\frac{a(\epsilon)}{4}$. Suppose that $p_{k} \in \omega_{\omega}\left(u_{k}\right)$, so we can find a subnet $\left\{u_{k}\left(t_{\alpha}\right)\right\}_{\alpha \in A}$ of $\left\{u_{k}(t)\right\}_{t \in G}$ with $\omega-\lim _{\alpha \in A} u_{k}\left(t_{\alpha}\right)=p_{k}$ in which, for every $\alpha \in A, t_{\alpha} \geq t_{0}$, in which $A$ is a directed system. Using Lemma 2.3, for every $p \in G_{a\left(a\left(\frac{a(\epsilon)}{16}\right)\right)}$, we can find $n \in \mathbb{N}$ such that, for every $\alpha \in A$,

$$
\frac{1}{n} \sum_{i=1}^{n} J_{k}\left(p^{i}\right) u_{k}\left(t_{\alpha}\right) \in F_{\frac{a(\epsilon)}{4}}\left(J_{k}(p)\right) .
$$


Since for each $\alpha \in A$

$$
\begin{aligned}
& \left\|\left(\frac{1}{n} \sum_{i=1}^{n} J_{1}\left(p^{i}\right) u_{1}\left(t_{\alpha}\right)-\frac{1}{n} \sum_{i=1}^{n} u_{1}\left(p^{i} t_{\alpha}\right), \ldots, \frac{1}{n} \sum_{i=1}^{n} J_{k}\left(p^{i}\right) u_{k}\left(t_{\alpha}\right)-\frac{1}{n} \sum_{i=1}^{n} u_{k}\left(p^{i} t_{\alpha}\right)\right)\right\|_{k} \\
& \quad \leq \varphi\left(t_{\alpha}\right)<\frac{a(\epsilon)}{4},
\end{aligned}
$$

we get

$$
\frac{1}{n} \sum_{i=1}^{n} u_{k}\left(p^{i} t_{\alpha}\right) \in \frac{1}{n} \sum_{i=1}^{n} u_{k}\left(p^{i} t_{\alpha}\right)+B\left(0, \frac{a(\epsilon)}{4}\right) \subset F_{a(\epsilon)}\left(J_{k}(p)\right) .
$$

Since $u_{k}(p t)-u_{k}(t) \rightarrow 0$ for every $p \in G$, we have $u_{k}\left(p^{i} t_{\alpha}\right) \rightarrow p_{k}, i=1,2, \ldots, n$. Then, for all $p \in G_{a\left(a\left(\frac{a(\epsilon)}{16}\right)\right)}$,

$$
p_{k}=\omega-\lim _{\alpha \in A} \frac{1}{n} \sum_{i=1}^{n} u_{k}\left(p^{i} t_{\alpha}\right) \in \overline{\mathrm{co}} F_{a(\epsilon)}\left(J_{k}(p)\right) .
$$

So, Lemma 2.1, implies that for every $p \in G_{a\left(a\left(\frac{a(\epsilon)}{16}\right)\right)}, p \in F_{\epsilon}(J(p))$, hence $p_{k} \in F\left(\Im_{k}\right)$.

In three last theorems $X$ has not a Frechet differentiable norm.

Theorem 2.8 Assume that $\left\{\left(X^{k},\|\cdot\|_{k}\right)\right\}_{k \in \mathbb{N}}$ is a uniformly convex multi-Banach space with the Kadec-Klee property for its dual, and $\emptyset \neq C \subset X$ is bounded and closed. Suppose that $\Im_{k}=\left\{J_{k}(t): t \in G\right\}$ of a reversible semigroup of asymptotically nonexpansive function on $C$ and $u_{k}(\cdot)$ is an almost orbit of $\Im_{k}$. Then the following statements are equivalent:

(1) $\omega_{\omega}\left(u_{k}\right) \subset F\left(\Im_{k}\right)$;

(2) $\omega-\lim _{t \in G} u_{k}(t)=p_{k} \in F\left(\Im_{k}\right)$;

(3) $\omega-\lim _{t \in G} u_{k}(p t)-u_{k}(t)=0$ for every $p \in G$.

Proof (1) $\Rightarrow$ (2). It is enough to prove that $\omega_{\omega}\left(u_{k}\right)$ is a singleton. The reflexivity of $X \mathrm{im}$ plies that $X \neq \emptyset$. Suppose that $\varphi_{k}$ and $g_{k}$ are two elements in $\omega_{\omega}\left(u_{k}\right)$, then by (1) we get $\varphi, g \in F\left(\Im_{k}\right)$. For every $\gamma \in(0,1)$, using Lemma 2.4, we have $\lim _{t \in G} \|\left(\gamma u_{1}(t)+(1-\gamma) \varphi-\right.$ $\left.g, \ldots, \gamma u_{k}(t)+(1-\gamma) \varphi-g\right) \|_{k}$ exists. Put

$$
h(\gamma)=\lim _{t \in G}\left\|\left(\gamma u_{1}(t)+(1-\gamma) \varphi-g, \ldots, \gamma u_{k}(t)+(1-\gamma) \varphi-g\right)\right\|_{k}
$$

then for given $\epsilon>0$, there is $t_{1} \in G$ such that, for every $t>t_{1}$,

$$
\left\|\left(\gamma u_{1}(t)+(1-\gamma) \varphi-g, \ldots, \gamma u_{k}(t)+(1-\gamma) \varphi-g\right)\right\|_{k} \leq h(\gamma)+\epsilon .
$$

So, for every $t \geq t_{1}$,

$$
\left\langle\gamma u_{k}(t)+(1-\gamma) \varphi-g, j(\varphi-g)\right\rangle \leq\|\varphi-g\|(h(\gamma)+\epsilon),
$$

in which $j(\varphi-g) \in J(\varphi-g)$. Let us note $\varphi \in \overline{\mathrm{co}}\left\{u_{k}(t): t \geq t_{1}\right\}$, so

$$
\langle\gamma \varphi+(1-\gamma) \varphi-g, j(\varphi-g)\rangle \leq\|\varphi-g\|(h(\gamma)+\epsilon),
$$


which means $\|\varphi-g\| \leq h(\gamma)+\epsilon$. We know $\epsilon$ is arbitrary, then

$$
\|\varphi-g\| \leq h(\gamma)
$$

$g \in \omega_{\omega}\left(u_{k}\right)$ implies that there is a subnet $\left\{u_{k}(t-\alpha)\right\}_{\alpha \in A}$ in $\left\{u_{k}(t)\right\}_{t \in G}$ such that $\omega$ - $\lim _{\alpha \in A} u_{k}\left(t_{\alpha}\right)=g$, in which $A$ is a directed system. Setting

$$
I=A \times \mathbb{N}=\{\beta=(\alpha, n): \alpha \in A, n \in \mathbb{N}\},
$$

then for $\beta_{i}=\left(\alpha_{i}, n_{i}\right), i \in I, i=1,2$, define $\beta_{1} \leq \beta_{2}$ iff $\alpha_{1} \leq \alpha_{2}, n_{1} \leq n_{2}$. For arbitrary $\beta=(\alpha, n) \in I$, define $P_{k, 1} \beta=\alpha, P_{k, 2} \beta=n, t_{\beta}=t_{\alpha}, \epsilon_{\beta}=\frac{1}{P_{k, 2} \beta}$. Then $\omega$ - $\lim _{\beta \in I} u_{k}\left(t_{\beta}\right)=g$ and $\lim _{\beta \in I} \epsilon_{\beta}=0$. Using Lemma 1.6 implies that

$$
\begin{aligned}
& \left\|\left(\gamma u_{1}\left(t_{\beta}\right)+(1-\gamma) \varphi-g, \ldots, \gamma u_{k}\left(t_{\beta}\right)+(1-\gamma) \varphi-g\right)\right\|_{k} \\
& \quad \leq\|\varphi-g\|^{2}+2 \gamma\left\langle u_{k}\left(t_{\beta}\right)-\varphi, j\left(\gamma u_{k}\left(t_{\beta}\right)+(1-\gamma) \varphi-g\right)\right\rangle .
\end{aligned}
$$

Using Lemma 2.4 and the inequality $\|\varphi-g\| \leq h(\gamma)$ implies that

$$
\liminf _{\beta \in I}\left\langle u_{k}\left(t_{\beta}-\varphi, j\left(\gamma u_{k}\left(t_{\beta}\right)+(1-\gamma) \varphi-g\right)\right\rangle \geq 0\right.
$$

So, for each $\xi \in I$, there is $\beta_{\xi} \in I$ such that $\beta_{\xi} \geq \gamma$ and

$$
\left\langle u_{k}\left(t_{\beta_{\xi}}\right)-\varphi, j\left(\epsilon_{\xi} u_{k}\left(t_{\beta_{\xi}}\right)+\left(1-\epsilon_{\xi} \varphi-g\right)\right)\right| \geq-\epsilon_{\xi} .
$$

It is well known that $\left\{\beta_{\xi}\right\}$ is also a subnet of $I$, then $\omega-\lim _{\xi \in I} u_{k}\left(t_{\beta_{\xi}}\right)=g$. Set

$$
j_{\xi}=j\left(\epsilon_{\xi} u_{k}\left(t_{\beta_{\xi}}\right)+\left(1-\epsilon_{\xi} \varphi-g\right)\right)
$$

The reflexivity of $X$ implies that $X^{*}$ is also reflexive, and therefore the set of all weak limit points of $\left\{j_{\xi}, \xi \in I\right\}$ is nonempty. Then, without loss of generality, let $\omega-\lim _{\xi \in I} j_{\xi}=j \in X^{*}$. Then $\|j\| \leq \liminf _{\xi \in I}\left\|j_{\xi}\right\|=\|\varphi-g\|$. Since

$$
\left\langle\varphi-g, j_{\xi}\right\rangle=\left\|\epsilon_{\xi} u_{k}\left(t_{\beta_{\xi}}+\left(1-\epsilon_{\xi}\right) \varphi-g\right)\right\|^{2}-\epsilon_{\xi}\left\langle u_{k}\left(t_{\beta_{\xi}}-\varphi, j_{\xi}\right\rangle .\right.
$$

Passing the limit for $\xi \in I$, we get $\langle\varphi-g, j\rangle=\|\varphi-g\|^{2}$, which implies $\|j\| \geq\|\varphi-g\|$. Then

$$
\langle\varphi-g, j\rangle=\|\varphi-g\|^{2}=\|j\|^{2}
$$

i.e., $j \in J(\varphi-g)$. Hence, $\omega-\lim _{\xi \in I} j_{\xi}=j$ and $\lim _{\xi \in I}\left\|j_{\xi}\right\|=\|j\|$. By the reflexivity of $X^{*}$ and the Kadec-Klee property, we conclude that $\lim _{\xi \in I} j_{\xi}=j$. Take the limit for $\xi \in I$ in 2.2 , we get $\langle g-\varphi, j\rangle \geq 0$, i.e., $\|\varphi-g\|^{2} \leq 0$, which implies $\varphi=g$.

(2) $\Rightarrow$ (3). Obviously.

(3) $\Rightarrow$ (1). See Theorem 2.7 . 
Funding

No funding was received.

Competing interests

The authors declare that they have no competing interests.

\section{Authors' contributions}

All authors conceived of the study, participated in its design and coordination, drafted the manuscript, participated in the sequence alignment, and read and approved the final manuscript.

\section{Author details}

'Science and Research Branch, IAU, Tehran, Iran. ${ }^{2}$ Department of Mathematics, Iran University of Science and Technology, Tehran, Iran. ${ }^{3}$ Research Institute for Natural Sciences, Hanyang University, Seoul, Republic of Korea.

\section{Publisher's Note}

Springer Nature remains neutral with regard to jurisdictional claims in published maps and institutional affiliations.

Received: 21 November 2018 Accepted: 11 February 2019 Published online: 21 February 2019

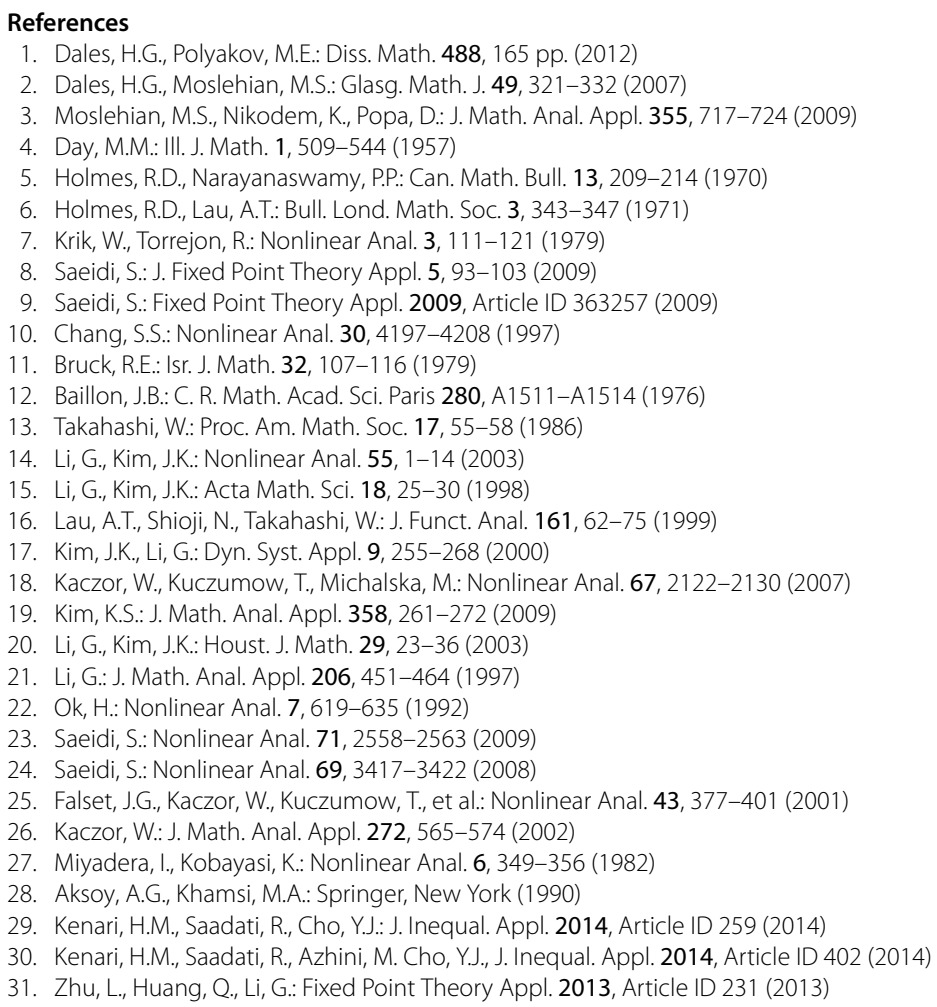

\section{Submit your manuscript to a SpringerOpen ${ }^{\circ}$ journal and benefit from:}

- Convenient online submission

Rigorous peer review

- Open access: articles freely available online

- High visibility within the field

- Retaining the copyright to your article

Submit your next manuscript at $\gg$ springeropen.com 